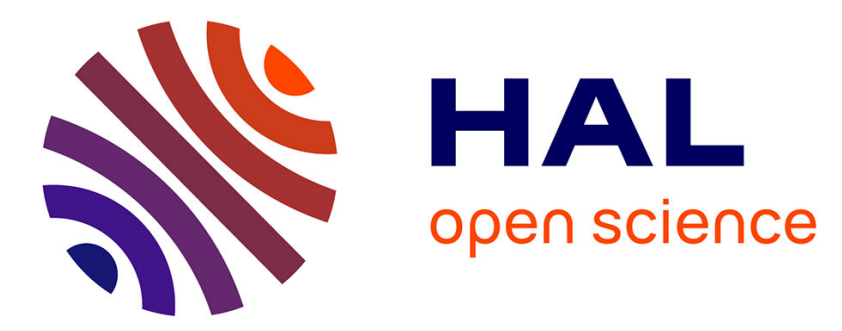

\title{
Les rats de la Saint-Jean. Une lecture ethnocritique d'Angèle \\ Jean-Marie Privat
}

\section{To cite this version:}

Jean-Marie Privat. Les rats de la Saint-Jean. Une lecture ethnocritique d'Angèle. Pratiques : linguistique, littérature, didactique, 1992, 76, pp.27-30. 10.3406/prati.1992.1676 . hal-03180247

\section{HAL Id: hal-03180247 \\ https://hal.univ-lorraine.fr/hal-03180247}

Submitted on 24 Mar 2021

HAL is a multi-disciplinary open access archive for the deposit and dissemination of scientific research documents, whether they are published or not. The documents may come from teaching and research institutions in France or abroad, or from public or private research centers.
L'archive ouverte pluridisciplinaire HAL, est destinée au dépôt et à la diffusion de documents scientifiques de niveau recherche, publiés ou non, émanant des établissements d'enseignement et de recherche français ou étrangers, des laboratoires publics ou privés. 


\section{Citer ce document / Cite this document :}

Privat Jean-Marie. Les rats de la Saint-Jean. Une lecture ethnocritique d'Angèle. In: Pratiques : linguistique, littérature, didactique, $n^{\circ} 76,1992$. pp. 27-30;

doi : https://doi.org/10.3406/prati.1992.1676

https://www.persee.fr/doc/prati_0338-2389_1992_num_76_1_1676

Fichier pdf généré le 13/07/2018 


\title{
LES RATS DE LA SAINT-JEAN UNE LECTURE ETHNOCRITIQUE D'ANGËLE
}

\author{
Jean-Marie PRIVAT
}

«Quand Angèle fut seule... » : la société villageoise traditionnelle conjugue en effet, structurellement, " enfermement du groupe domestique sur lui-même et ouverture sur la communauté toute entière, intensité des rapports intrafamiliaux et vivacité des liens communautaires $»(1)$.

C'est dans cette tension dialectique, vécue tragiquement, entre le pôle public et le pôle privé qu'Angèle va affirmer « sa dignité ".

\section{L'espace local des relations communautaires}

Le village est un cadre de vie, stricto sensu. Baptiste et Angèle ne sont " jamais sortis de Sainte-Croix », sans grand regret d'ailleurs; et par deux fois Baptiste supplie sa femme de ne pas « les » laisser l'emmener à l'hôpital. Mourir chez soi, entre soi, est tout à fait dans la logique localiste des existences coutumières micro-socialisées.

Cette «territorialité " tout à la fois culturelle, culturale (semis de radis, saulaie, pommiers, champs, vigne) et cultuelle (cimetière, église, curé, SainteCroix) est d'ordinaire structurée selon un système de relations d'aides et d'entraide communautaires, mais aussi tramée de contraintes et de contrôles publics et collectifs.

L'inter-dépendance et l'inter-connaissance généralisées, permanentes et réciproques, sont ainsi les traits caractèristiques de la sociabilité villageoise. Aussi, Baptiste connaît-il « bien trop » le médecin pour s'inquiéter outre mesure de sa visite ; aussi l'église est-elle "pleine » pour l'enterrement. D'ailleurs, Angèle aurait pu citer «le nom de tous ceux qui étaient là ».

(1) F. Zonabend, La Mémoire longue, Paris, P.U.F., 1980. 
La participation de la communauté se manifeste donc avec force dans les moments de crise, de « rupture » : même la vieille Thibault « qu'on n'avait pas vue depuis un an au moins " était là, et même Germaine Richard, la catin.

Cette participation effective est aussi une coopération affective au travail de deuil : « on » lui avait " souvent » dit que le « retour du cimetière » est le moment «le plus pénible ». L'ethnologue de la France traditionnelle rappelle que la mort, en tant qu' « événement social " particulièrement dramatique, sollicite directement les services du voisinage qui accomplit ainsi son "devoir social » (2), ni plus ni moins. Les chaises, «serrées en rang d'oignon le long du lit », témoignent effectivement d'une veillée mortuaire collective et Cécile, en bonne voisine, " après s'être changée " était venue voir si la veuve solitaire " n'avait besoin de rien ». Et sa conversation, comme il se doit, rappelle les "larmes de quelques-uns", "le chagrin de tous".

Inversement, ne pas être connu ou ne pas être reconnu, c'est tout un. Le fils Richard, "dont personne n'avait rien su ", était probablement un bâtard : "on ne le connaisait pas à Sainte-Croix ».

Etre du village permet donc de jouir des solidarités locales mais, en contrepartie, exige d'en accepter les règles, les normes et les valeurs (3).

Les rites cérémoniels, garants de l'intégrité du groupe, sont intangibles : «il lui avait fallu se faire embrasser par tout le village ». Et c'est dans la certitutde paisible d'une corvée achevée et du rite accompli que se fait le retour à la maison : "Tout s'était bien passé ».

A Sainte-Croix, comme dans tous les petits groupes à base locale, "les individus vivent presque totalement sous le regard les uns des autres ", c'està-dire que " tout le monde contrôle tout le monde » (4). Cécile était donc dans son rôle quand, venant tenir compagnie à Angèle, elle lui apportait les " dernières nouvelles du village ", se risquant même à lui dire, un jour, "qu'il lui semblait avoir aperçu Baptiste discutant avec Germaine, près de la vigne »...

\section{Les rites du temps}

Les identités locales et individuelles s'ancrent aussi dans un temps partagé et accepté dont chacun est à la fois l'héritier et le garant.

Angèle se souvient par exemple qu'André, toujours là, « lui faisait tourner la tête, au bal, il y a bien quarante ans de cela »; Baptiste, lui, qui disait « tenir sa vieille bouffarde de son père, qui lui-même... » sait et reconnaît qu'il " a fait son temps».

Le temps de la routine quotidienne («Pas besoin de montre. Elle savait quand il lui fallait aller nourrir les volailles, préparer le dîner. Elle savait quand Baptiste rentrait ") est rythmé par un temps rituel, calendaire ou non. Le cycle des rites funéraires par exemple ne consiste pas seulement à enterrer, cérémoniellement, le mort. II faut encore que « la mort quitte la maison» (5). Aussi Angèle éloigne-

(2) A. Van Gennep, Manuel de folklore français contemporain, tome premier, II, Du berceau à la tombe (fin), mariage-funérailles, Paris, éd. Picard, 1946, (rééd.,1976).

(3) Voir pour un exemple qui touche une héroïne romanesque célébre, J.-M. Privat, "Essai d'ethnologie du texte littéraire : les charivaris dans Madame Bovary ", Ethnologie française, 1988, 3.

(4) P. Bourdieu, «Célibat et condition paysanne », Etudes rurales, 5-6, 1962.

(5) A. Van Gennep, op.cit. 
t-elle de la chambre mortuaire, pour accomplir le rite, les «bougies » d'abord, les « chaises » ensuite, le «balai » enfin.

Dans cet univers villageois, les cérémonies périodiques et saisonnières ont, bien évidemment, toute leur importance. Ainsi Baptiste aurait-il «bien voulu tenir jusqu'à la Saint-Jean » pour que la boucle soit bouclée, pour que son destin s'inscrive, par une heureuse coïncidence, dans le cycle des rites collectifs. II n'avait d'ailleurs même pas besoin de le dire car Angèle aussi « le savait ».

Si cela suffisait à le contenter c'est peut-être aussi parce que, même au plus fort de la douleur, il soutenait volontiers qu'il allait mieux et que bientôt, sans doute, tout cela serait "oublié ». Depuis le temps (vingt ans) que son mal le " taquinait ", il s'était " habitué ", d'une Saint-Jean à l'autre, à durer, quelque incompréhension qu'en ait son médecin...

Or, Baptiste ne pouvait guère ignorer que « la veille, la nuit et le jour de la Saint-Jean sont une de ces périodes fastes ou néfastes regardées populairement comme possédant certaines vertus, utiles ou dangereuses » (6). Baptiste le bien nommé savait que, comme il est de coutume la nuit de la Saint-Jean, avant le lever du soleil, "à la fine pointe de l'aube », pucelles et vieilles femmes de préférence, cueillent ces herbes merveilleuses qui, entre autres, "guérissent les maux d'estomac » (7) et éloignent les mauvais sorts.

II connaissait forcément, comme tout villageois, cette croyance et cette pratique magique qui veut que jeunes gens et jeunes filles à marier sautent par-dessus les feux des bûchers solsticiaux pour s'assurer d'un mariage fécond!

On aura noté bien sûr que Baptiste meurt très précisément « au petit jour ", en ce moment quasi sacré où le temps doit «passer ». Angèle de son côté, avait aimé "son » Baptiste, " dés le début, ou presque » : ne l'accompagnait-elle pas " aux champs » pour "lui donner la main »? Angèle "savait » que son mari la trompait et Angèle « savait » que Baptiste attendait la Saint-Jean... Peut-être avait-elle esssayé, en vain, les recettes magiques des féminines Evangiles des Quenouilles : "Pour estre de son mary amée, il faut lui faire mangier une poirée d'herbes cueillies la nuit Sainct Jehan, a nonne, et pour vray, il ne lui seroit possible de la laissier pour une aultre plus jone... » (8) !

Mais il est vrai aussi que dans le bestiaire érotico-folklorique des villages on appelle communèment « rat » celui qui fait montre d'une sexualité nomade et féconde. Aussi Angèle pouvait-elle penser dans son for intérieur qu'il ne « suffit » pas, comme le veut la tradition en Beauce, en Touraine ou dans le Pays de Caux, " de jeter avec une cuiller de bois, un peu d'eau rosée, recueillie avant le lever du soleil, sur les tas de blés ou dans les vignes, le jour de la Saint-Jean, pour éloigner les rats » (9). Elle avait pu observer, par contre, depuis vingt ans, l'efficacité lentement redoutable de la mort-aux-rats. Le « mal » avait donc triomphé. C'était « normal ». Maintenant que le « rat » était enterré, en effet, la mort (aux-rats) pouvait quitter la maison...

(6) A. Van Gennep, op. cit., tome premier, vol. 4, Cérémonies périodiques cycliques et saisonnières, 2-cycle de mai, cycle de saint Jean et saint Pierre, Paris, éd. Picard, 1949 (rééd., 1981).

id., ibid.

(8) Anonyme, Les Evangiles des Quenouilles, Paris, Jannet, 1855 (première édition connue en 1475)

(9) A. Van Gennep, op.cit. 


\section{L'ange du mal(e)}

Il y a de la sorcière maléfique dans cette femme, qui a su attendre son heure. Baptiste, pris au piège des maux / mots, fait comme un rat, s'abandonne sans méfiance à son ange gardien à la patience d'ange : vingt ans durant, " du coin de l'œil » Angèle surveille le café, quotidiennement fatidique, de Baptiste. Autrement dit, pour être stérile, Angèle n'en est pas pour autant... angélique.

C'est dire si l'univers villageois, clos, apparemment stable et ordonné, est en fait parcouru de tensions latentes, clivé par des conflits secrets (et qu'il secréte) : en témoignent l'inféconde et diabolique Angèle, Baptiste le « rat », Germaine la catin.

Le code dominant de la morale sexuelle et / ou conjugale est lui-même si organiquement constitutif de la (re-)production du groupe que toute transgression personnelle est interdite, condammnée à être occultée ou exposée à être spectaculairement châtiée.

Mais, manifestement, Angèle refuse de s'inscrire aussi bien dans la logique étatique moderne (divorcer au tribunal) que dans la logique coutumière ancienne (donner droit aux rituelles conduites vindicatives populaires : promenades sur l'âne, charivari, jugement carnavalesque) : « elle avait décidé de ne jamais en parler à Baptiste, ni à personne ».

II ne lui reste même pas la place socio-historiquement marginale et tragique de la sorcière de village qui peut, au moins, protester publiquement de sa " dignité » bafouée et affirmer un « pouvoir ».

Pour sortir de sa contradiction - s'affirmer (selon une logique " bourgeoise" d'individuation) dans un tissu de relations instituées (selon une logique « populaire » d'affiliation) - elle est contrainte à un double je / jeu meurtrier. Angèle donne le change en se comportant «comme il faut », exactement comme la narration qui, dans le codage onomastique par exemple, semble obéir aux conventions scripturales les plus transparentes (Baptiste voué à la Saint-Jean, Angèle vouée à (porter sa) Sainte-Croix, etc.) mais qui est en fait à retourner, à inverser purement et simplement.

Le propre de l'ethno-critique est donc de (re)construire une cohérence ethnologique du texte fictionnel et ainsi d'initier au relativisme textuel mais aussi de montrer comment des traits culturels populaires et folklorico-rituels sont réinterprétés du fait même de "leur insertion dans le système de relations constitutif de l'œuvre » (10).

Du point de l'anthropologie de la culture, l'ethno-critique met enfin en évidence que la loi du texte moderne, en (d)énonçant ici Angèle par exemple, est peut-être de médiatiser le texte de la Loi... (11)

(10) P. Bourdieu, «Lecture, lecteurs, lettrés, littérature », Choses dites, Paris, éd. de Minuit, 1987.

(11) Pour approfondir ces derniers points, voir J.-M. Privat, " Madame Bovary, mœurs de province de G. Flaubert : une lecture ethno-critique ", thèse de doctorat, Lyon 2, 1991 ou J.-M. Privat, "Le Carnaval de Boule-deSuif ", communication au colloque "Mémoire historique et récit populaire dans la seconde moitié du XIX" siécle ", Lyon, 1992, à paraître. 